\title{
On the modelling of residual stresses due to surface peening using eigenstrain distributions
}

\author{
A M Korsunsky \\ Department of Engineering Science, University of Oxford, Parks Road, Oxford OX1 3PJ, UK. \\ email: alexander.korsunsky@eng.ox.ac.uk
}

The manuscript was received on 09 February 2005 and was accepted after revision for publication on 08 July 2005.

DOI: $10.1243 / 030932405 X 30984$

\begin{abstract}
The beneficial effect of surface peening (including shot peening, laser shock peening, low plasticity burnishing, etc.) on fatigue resistance of structural alloys is well documented and widely used in industry to improve component life. It is perhaps the most widely used technique that relies on the introduction of residual stresses into the component by direct mechanical means. The resulting stress states are usually evaluated experimentally by X-ray diffraction or other stress measurement techniques. In order for shot peening operations to be effective and reliable, the process of introducing residual stresses and strains into peened components must not only be controlled but also be predictable and quantifiable.

The present paper establishes a framework for predictive modelling of the residual stress states due to surface peening. Firstly, uniform (within planes parallel to the surface) plastic deformation of the surface layer taking place during shot peening of a thin plate is simulated using the concept of eigenstrain.

As a further demonstration of the capabilities of the proposed approach, flexible plate theory is applied to the analysis of stresses and deformations arising due to local peening within a circular area. The results are of considerable use and significance for the analysis of shot peening and peen forming operations.
\end{abstract}

Keywords: residual stresses, surface peening, eigenstrain distributions, modelling

\section{INTRODUCTION}

Shot peening is a cold working process aimed at creating substantial (of the order of 60 per cent of the material yield strength) levels of compressive residual stress in a thin near-surface layer of material. This is achieved by bombarding the metal surface with spherical particles, such as steel or ceramic shot or glass beads, under controlled conditions. Success in preventing, or substantially retarding stress corrosion cracking, crack initiation and growth, pitting, fretting, and other fatigue phenomena, by shot peening has been reported for various steels, as well as for aluminium and titanium alloys, the Inconels, copper silicon alloys, and other materials [1]. The mechanism of fatigue retardation is associated in part with work hardening of the surface, but is mainly due to the plastic strain, which puts the outer layers of material into compression.

A somewhat arbitrary distinction is sometimes made between two possible modes of plastic deformation [2], namely one dominated by stretching (hard shot on softer substrate) or by subsurface compression (soft shot on harder substrate). Guechichi et al. [3] have developed a cyclic loading model that predicts depth distribution of shot peening stresses in a semi-infinite solid based on the Hertzian elastic contact solution, and the cyclic elastic limit of the substrate material. Perhaps one of the more important conclusions is that to good accuracy the plastic strain can be assumed constant to a certain characteristic penetration depth, and zero elsewhere.

In an application of the shot peening treatment, the predictions for residual stresses obtained using the above approach must be adjusted to specific finite geometries. The key to better integration of shot peening into the design process lies in the ability to calculate reliably the increase in allowable stress. At present this approach can very rarely be implemented, with extensive fatigue testing being used instead at early stages of design. The primary concern in the practical application of shot peening is repeatability. 
This is achieved by careful monitoring of the peening conditions, the Almen intensity being the primary parameter. The intensity is an indication of the kinetic energy transferred during the peening process. The Almen gauge is a standard measurement device for monitoring the arc height of a strip of material after its exposure to a blast of shot.

The model presented here is based on the author's earlier studies [4]. It provides a more general framework than that based on the 'stress source' solution of Flavenot and Niku-Lari [5]. The present paper addresses the question of elastic stress redistribution in finite thickness specimens after the introduction of permanent plastic strains by shot peening, and is related to the interpretation of Almen intensity and peen forming of thin plates. The approach described in the present paper starts with an assumed distribution of permanent plastic strain introduced into the workpiece by the peening process and proceeds to derive the residual stress state that arises in response, under the beam or shell bending approximation. It is therefore important to note that peen process modelling is not part of the proposed routine. The residual stress is thought to arise due to elastic stress equilibration and associated plate bending. An analytical framework incorporating shot peening effects into the flexible plate theory is presented and its application to model problems is considered.

A significant number of publications can be found in the literature dedicated to the modelling of the peen process and of the resulting residual stress state. Kobayashi et al. [6] consider the popular approach based on the study of plastic deformation induced by the impact of individual shot. They review the literature dedicated to shot peen modelling and observe that a common difficulty arising in this approach lies in the fact that simulating an extremely large number of complex dynamic processes associated with each impact involved in the peening process is computationally prohibitively expensive. The authors conclude that experimental characterization of residual stress states after shot peening remains a very important requirement.

Blodgett and Nagy [7] describe their results obtained using one of the methods of experimental residual stress characterization that uses eddy current measurements. They also discuss the use of X-ray diffraction for the purpose of residual stress evaluation. An overview of laboratory-based X-ray methods is given by Hauk [8], while Prevey (e.g. see reference [9]) published a large number of experimental results for various systems and surface treatment methods, including low plasticity burnishing, laser shock peening, gravity peening, etc.

Wang and Platts $[\mathbf{1 0}]$ focus their attention on the deformation induced in thin-walled components by the peening process and propose a finite element framework for modelling peen forming based on the bending theory. Levers and Prior [11] present an approach to peening-induced residual stress modelling that relies on experimentally determined plastic strain distributions with depth from the surface, and use the finite element framework in order to predict the distortion (peen forming effect) arising from the treatment, using thermal strains for their simulation. A comparison of the present method with the study of Levers and Prior is provided below.

In the approach adopted in the present study it is the induced plastic strain distribution that is considered to be the primary characteristic of the peening process. Clearly, this distribution stands in a complex relationship to the material properties, shot size, hardness, speed and angle of impact, and also workpiece dimensions. Plastic deformation within the workpiece arises due to a local stress increase under impact. At large distances from the impact site the contact-induced stresses decay inversely in proportion to the distance. It is therefore reasonable to assume that, as the size of the workpiece increases, the influence of its dimensions on the interaction between a shot and the surface becomes less and less significant, and for large workpieces the resulting plastic strain distribution becomes independent of the workpiece thickness. Although this hypothesis is not employed for the analysis presented in this paper, it may provide a convenient basis for justifying the use of plastic strain distribution as a process characterization parameter, and deserves experimental validation.

A further issue in the analysis of fatigue life improvement afforded by surface peening is the incorporation of residual stress into the numerical model for cyclic elastoplastic deformation and the predictive model for crack initiation. Guagliano and Vergani [12] discuss the influence of the peeninginduced residual stress state on crack growth in engineering components, using a procedure for applying an experimentally determined residual stress state to the crack surfaces within the finite element framework.

It is worth noting here that, under fatigue conditions, the presence of residual stress is not simply equivalent to a modification of the mean stress. Firstly, residual stress modifies local yielding conditions, thus affecting both the stress and strain range, and the mean stress. Secondly, the residual stress itself changes during cyclic plastic deformation. This happens as a result of the generation of additional inelastic strain, or eigenstrain. Furthermore, the growth of cracks is likely to cause stress redistribution, which in turn affects the conditions of crack propagation. The concept of eigenstrain provides a rational and rigorous basis for the incorporation of residual stress into deformation modelling. 
To ensure clarity, it is convenient to split the problem into several stages. In this paper the only part of the problem addressed concerns the determination of the residual stress state given a continuous eigenstrain distribution. This may be called the 'direct problem'. In practice the problem is quite often more difficult and may be formulated as follows: residual elastic strains (or their changes due to machining) have been measured in a particular geometry at a finite number of points (or averaged across gauge volumes). The underlying eigenstrain distribution responsible for the residual stress state must be found. This problem is the inverse problem of eigenstrain analysis. It can be addressed efficiently for arbitrary geometries using a variational approach, described in a separate study. In the present article only the direct problem is discussed.

It is worthwhile here to make a brief note regarding the role of the concept of eigenstrain in residual stress analysis. Eigenstrain can be thought of as arbitrary permanent inelastic strain introduced into the object by a process other than elastic deformation. This could be associated with time-independent plasticity (as in the case of shot peening), creep, transformation-induced strain, welding, or even a cutting and pasting operation.

The last operation noted in the above list is particularly relevant in order to appreciate the eigenstrain concept. It is instructive to perform a thought experiment whereby an annulus of elastic material is cut along its radius so that some of the material along the cut line is lost (Fig. 1). Now let the annulus be glued back together. Since the annulus no longer fits together neatly (is 'misfitting'), this operation will induce a residual stress state in it. The origin of this residual stress state is eigenstrain, which could be conveniently associated with the location of the cut. Note that the term 'eigenstrain' is being used here even though no deformation (strain) has taken place in connection with the cutting and pasting operation. It is useful to imagine that the same final state could be achieved without cutting, but instead by imagining that the material lost in the process of cutting had suddenly undergone a transformation process whereby its hoop extent has diminished to zero.

Eigenstrain can also be thought of as the perturbation imposed upon the theory of elasticity formulation. The conventional elastic problem formulation (in terms of strains or stresses) consists of equations of stress equilibrium, strain compatibility, and the elastic stress-strain relations. Deviations from this framework (e.g. plasticity) are usually thought of as modifications to the latter part of the formulation, i.e. non-linear stress-strain relations. It is proposed here to consider a different view, namely that eigenstrain gives rise to a non-zero right-hand side of the equations of strain compatibility (more appropriately,

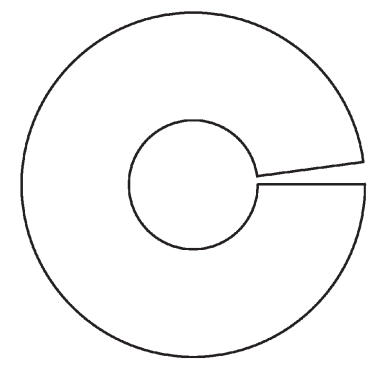

(a)

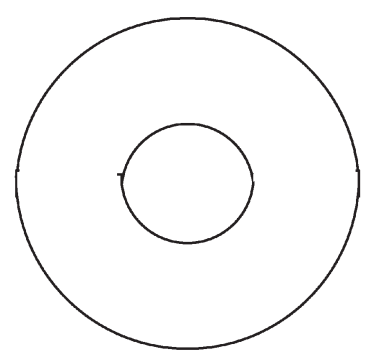

(b)
Fig. 1 (a) Illustration of an annular specimen with material cut-out that is equivalent to a localized eigenstrain. (b) After the eigenstrain is introduced, the annulus contains residual stress and may undergo distortion (depending on the nature of the eigenstrain introduced)

incompatibility). The evolution of eigenstrain during inelastic deformation processes (cutting and pasting, creep, plastic deformation, etc.) is a subject external to the elastic formulation and must be described by empirical equations of state.

In the analysis of residual stress it is not necessary to know anything about the origin of eigenstrain, but only about its spatial extent and variation. Once eigenstrain is prescribed, the residual stress state is found as the solution of a perturbed elastic problem, where eigenstrain appears as the perturbation of strain compatibility. If further inelastic processes were to take place, then the eigenstrain distribution becomes modified and a new residual stress is established. This view is particularly advantageous if such subjects need to be tackled as stress relaxation or object distortion during subsequent machining. The difficulty arising in such situations primarily stems from the complex geometry of real components that need to be considered. This usually precludes the use of analytical solutions and requires the application of numerical approaches, such as the finite element method. The use of variational eigenstrain modelling for such problems has been proposed and developed by the author, and will be reported in subsequent publications.

In the context of the present study the eigenstrain considered is associated purely with the plastic deformation induced by peen shot impact. The nature of the plastic strain tensor and its spatial variation are discussed. The perturbed elastic problem is then solved using two different approaches, for the cases of uniform (in two coordinates) and non-uniform (non-zero within a finite region) eigenstrain.

\section{UNIFORM EIGENSTRAIN}

In the first instance a plate is considered of thickness $h$ in the $z$ direction which contains uniformly distributed 


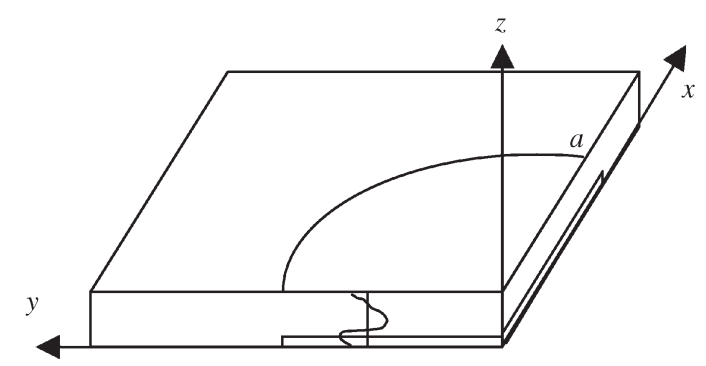

Fig. 2 Illustration of the problem geometry. The plate is infinitely extended in directions $x$ and $y$, and possesses thickness $h$ in direction $z$. In the uniform eigenstrain problem it is assumed that the eigenstrain domain is infinitely extended in directions $x$ and $y$, but varies in direction $z$ through the thickness of the plate. In the non-uniform eigenstrain problem it is assumed that the eigenstrain domain is also bounded in the plane parallel to the plate surface, and only exists within the circular domain $x^{2}+y^{2}<a^{2}$, as indicated in the figure

permanent plastic strain induced by peening (Fig. 2). Due to inherent symmetry of the shot peening operation under the conditions of normal impact, the eigenstrains $\varepsilon_{x}^{*}$ and $\varepsilon_{y}^{*}$ can be assumed equal throughout the analysis. In the present case they are also independent of the $x$ and $y$ coordinates, while their variation in the $z$ direction is assumed to be known, $\varepsilon_{x}^{*}=\varepsilon_{y}^{*}=\gamma(z)$. The third normal eigenstrain component $\varepsilon_{z z}^{*}$ must be constrained to be equal to $-2 \gamma(z)$, by virtue of volume conservation under the conditions of plastic deformation that generated it, while all shear eigenstrain components are assumed zero.

For a given function $\gamma(z)$, the stresses in the plate $\sigma_{x}$ and $\sigma_{y}$ are to be determined. Firstly, the depth of the shot peening affected zone, $d<h$, is defined as the maximum depth for which non-zero eigenstrains exist, i.e. $\gamma(z) \geqslant 0$. Then the following moments of this eigenstrain distribution can be defined as

$$
\Gamma=\frac{1}{h} \int_{0}^{d} \gamma(z) \mathrm{d} z, \quad \Gamma_{1}=\frac{1}{h^{2}} \int_{0}^{d} \gamma(z) z \mathrm{~d} z
$$

where $\Gamma$ may be called the depth-average eigenstrain, while $\Gamma_{1}$ is the eigenstrain moment.

Let us now show that the stress $\sigma=\sigma_{x}=\sigma_{y}$ in the plate is linearly dependent on the moments $\Gamma$ and $\Gamma_{1}$, and the eigenstrain distribution function $\gamma(z)$. Indeed, the biaxial stress $\sigma$ is related to the biaxial elastic strain $e$ via $\sigma=E e /(1-\nu)$, where $E$ and $\nu$ are the Young's modulus and Poisson's ratio of the material respectively. Here the usual Kirchhof bending theory is used. According to the Kirchhof hypothesis of straight normals, during bending the material points that originally lie on a normal, i.e. on a line perpendicular to the plate mid-plane, remain in a straight line, which only suffers a rotation due to bending. A consequence of this assumption is that the displacements, and hence the total strain must vary linearly through the plate thickness, i.e. $\varepsilon(z)=b+a z / h$. Here the total strain must be thought of as the sum of elastic and inelastic (i.e. plastic, eigen) strain. The elastic strain may be obtained by subtracting the eigenstrain from this total strain expression, so that the stress is given by

$$
\sigma=\frac{E}{1-\nu}\left[b+\frac{a z}{h}-\gamma(z)\right]
$$

By applying the static equilibrium requirements the unknown constants $a$ and $b$ can be determined as

$$
a=6\left(2 \Gamma_{1}-\Gamma\right), \quad b=2\left(2 \Gamma-3 \Gamma_{1}\right)
$$

These results may be recast in terms of the plate curvature radius $R$ and the neutral axis position $z_{0}$ as

$$
R=\frac{1}{2 \Gamma_{1}-\Gamma} \frac{h}{6}, \quad z_{0}=\frac{3 \Gamma_{1}-2 \Gamma}{2 \Gamma_{1}-\Gamma} \frac{h}{3}
$$

Finally, the expression for the stress is

$$
\sigma(z)=\frac{E}{1-\nu}\left[2 \Gamma\left(2-\frac{3 z}{h}\right)-6 \Gamma_{1}\left(1-\frac{2 z}{h}\right)-\gamma(z)\right]
$$

The relationship between the eigenstrain profile and the residual stress in this case is illustrated (in a normalized form) in Fig. 3. It is apparent that the eigenstrain influence on residual stress can be thought to consist of two effects. Firstly, the presence of eigenstrain causes the generation of a local proportional residual stress (with a coefficient equal to the Young's modulus) of opposite sign. Secondly, the material responds to the appearance of the local residual stress by global re-equilibration. In the present case this amounts simply to bending, which can be clearly identified in the figure. The difference between the complete residual stress profile and the continuation of the straight line representing bending gives a local residual stress component that is proportional and opposite in sign to the eiegenstrain.

It is worth noting the limiting case of $z / h$ being very small, i.e. the stress immediately below the shotpeened surface, which is given by

$$
\sigma(0)=-\frac{E}{1-\nu}\left[\gamma(0)+6 \Gamma_{1}-4 \Gamma\right]
$$

If the plate is very thick (compared to the depth of the shot peen affected zone, $d$ ), then the average eigenstrain $\Gamma$ and eigenstrain $\Gamma_{1}$ become very small, with the stress being given by

$$
\sigma(z)=-\frac{E}{1-\nu} \gamma(z)
$$

This important conclusion indicates that residual stress measured in uniformly peened massive 


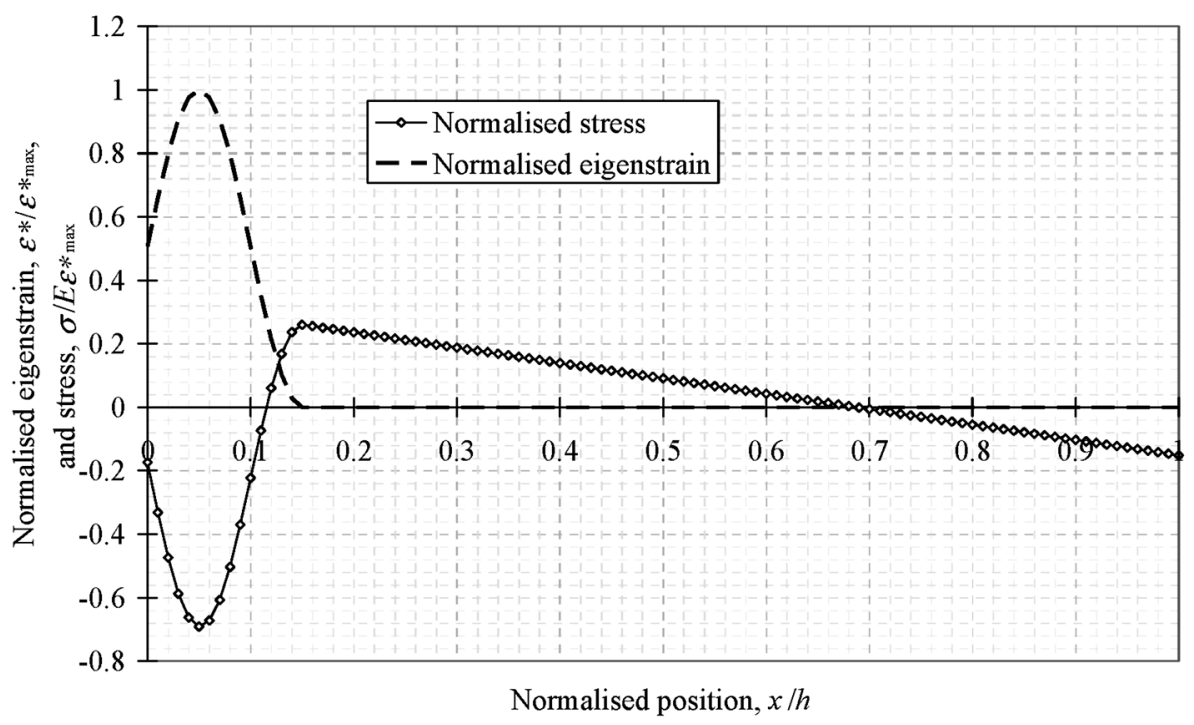

Fig. 3 An illustration of eigenstrain distribution (dashed line) and residual stress for the case of eigenstrain uniform in $x, y$. The horizontal axis is normalized by the total plate thickness, $h$. The vertical axis is normalized by the maximum value of eigenstrain, $E \varepsilon_{\max }^{*}$, for eigenstrain, and by Young's modulus times eigenstrain for residual stress, $E \varepsilon_{\max }^{*}$, for stress

workpieces is directly proportional to the permanent plastic strain introduced into the material. The result of this section, however, clearly demonstrates that this is not true for workpieces of finite geometry. Moreover, generally not one (e.g. Almen intensity) but two parameters, $\Gamma$ and $\Gamma_{1}$, may be required in order to provide an accurate description of the stress variation in plates of finite thickness.

The approach developed in the present paper is in agreement with other shot peen modelling results found in the literature. As an example consider the residual stress profile given by Levers and Prior [11] and shown in Fig. 4. Given that this distribution

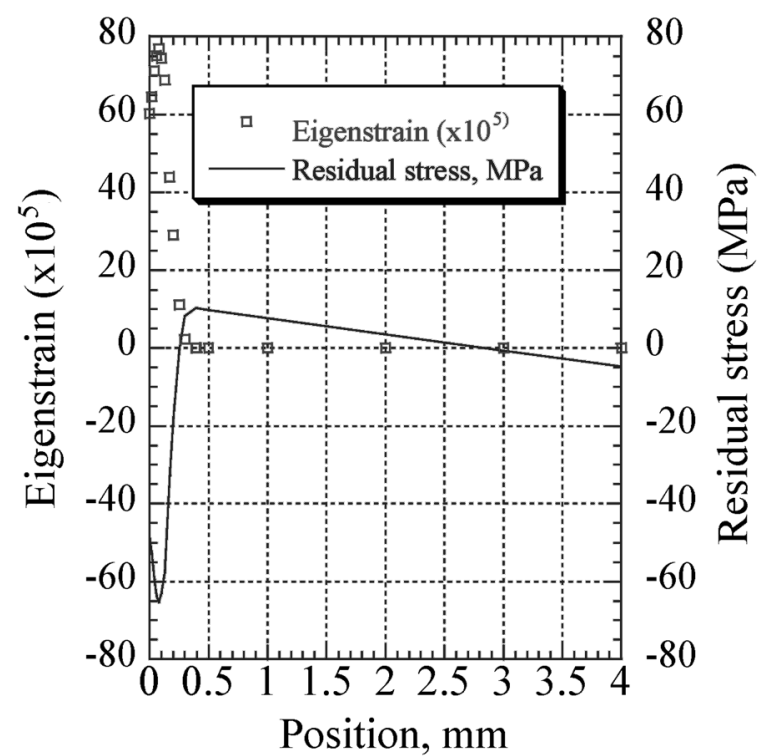

Fig. 4 Residual stress prediction compared with the results of Levers and Prior [11] arises in thick coupons and using the last equation above, the eigenstrain distribution can be obtained simply by dividing this profile by the value $E /(1-\nu)=103 \mathrm{GPa}$ approximately for the aluminium alloy considered. This corresponds to the maximum eigenstrain value of about $0.77 \times 10^{-3}$, or 770 microstrain. The residual stress can now be computed using the above formulae for the component shell thickness of $4 \mathrm{~mm}$ given in the paper, and is also shown in Fig. 4.

An elastic stress redistribution may similarly be expected to take place when spatially variant eigenstrains are introduced, as shown in the next section.

\section{NON-UNIFORM EIGENSTRAINS}

In this section the main steps are presented in the analysis of residual stresses due to spatially variant eigenstrains in a thin flexible plate. More specifically, a case of axisymmetric deformation due to the presence of eigenstrains inside a bounded domain $\Omega$ is considered. This simplification is chosen so that a closed-form solution can be derived. For the same reason, attention here will be confined to the case of uniform eigenstrains inside a domain of simple shape.

The present situation is a special case of a class of problems about inclusions containing eigenstrains. An example of a general method of formulation is given by Mura [13]. The problem when $\Omega$ is an ellipsoid in an infinitely extended homogeneous elastic solid and the eigenstrains are uniform was first solved in closed form by Eshelby [14]. Numerous applications of his famous result include crack problems, 
inhomogeneities, modelling of composite properties, etc. Inclusions of other shapes and other geometries have been considered. For example, the case of cuboidal inclusion was treated by Faivre [15], while a method of solution for a spheroidal inclusion inside one of two dissimilar elastic half-spaces has been considered by the present author [16].

A method of widest general applicability used in the derivation of elastic fields of known eigenstrain distributions is the method of Green's functions [13]. In the context of the present problem, however, it appears more convenient to proceed by a different route. The derivation of flexible plate equations will follow and the prescribed eigenstrains will be directly incorporated in the compatibility conditions.

Kirchhof's formulae for strains in a flexible plate (i.e. a plate for which both membrane and bending terms are significant) are given by

$$
\varepsilon_{r}=u_{, r}+\frac{1}{2}\left(\frac{\mathrm{d} w}{\mathrm{~d} r}\right)^{2}-z \frac{\mathrm{d}^{2} w}{\mathrm{~d} r^{2}}, \quad \varepsilon_{\theta}=\frac{u}{r}, \quad \gamma_{r \theta}=0
$$

Here $w$ is the vertical deflection and $r$ denotes the radial coordinate. When $z=0$ these expressions describe the deformation of the median plane. The equation of force equilibrium in the radial direction has the form

$$
\frac{\mathrm{d}}{\mathrm{d} r}\left(r S_{r}\right)-S_{\theta}=0
$$

where $S_{r}, S_{\theta}$ are the average stresses acting on elemental surfaces spanning the plate thickness $h$ with the normal orientated in the radial or hoop direction respectively. These are obtained by integration

$$
S_{r}=\frac{1}{h} \int_{0}^{h} \sigma_{r} \mathrm{~d} z, \quad S_{\theta}=\frac{1}{h} \int_{0}^{h} \sigma_{\theta} \mathrm{d} z
$$

and can be expressed in terms of the Airy stress function $\phi(r)$ introduced by $S_{r}=(1 / r)(\mathrm{d} \phi / \mathrm{d} r)$, $S_{\theta}=\left(\mathrm{d}^{2} \phi / \mathrm{d} r^{2}\right)$. The depth-averaged strains are then given by

$$
E \bar{\varepsilon}_{r}=\frac{1}{r} \frac{\mathrm{d} \phi}{\mathrm{d} r}-\nu \frac{\mathrm{d}^{2} \phi}{\mathrm{d} r^{2}}, \quad E \bar{\varepsilon}_{\theta}=\frac{\mathrm{d}^{2} \phi}{\mathrm{d} r^{2}}-\nu \frac{1}{r} \frac{\mathrm{d} \phi}{\mathrm{d} r}
$$

In the plane case considered, Saint-Venant's compatibility conditions are reduced to the single relation. Assuming the deflection $w$ is small, the corresponding term can be discarded, so that after averaging over the plate thickness this equation becomes

$$
\begin{aligned}
E\left[\frac{\mathrm{d}}{\mathrm{d} r}\left(r \bar{\varepsilon}_{\theta}\right)-\bar{\varepsilon}_{r}\right] & =r \frac{\mathrm{d}}{\mathrm{d} r} \nabla^{2} \phi \\
& =\frac{E}{h} \int_{0}^{h}\left[\varepsilon_{r}^{*}-\frac{\mathrm{d}}{\mathrm{d} r}\left(r \varepsilon_{\theta}^{*}\right)\right] \mathrm{d} z
\end{aligned}
$$

This is the equation for determining the stresses due to any given axially symmetric distribution of eigenstrains. Note that only the depth-averaged eigenstrains determine the total stress transmitted through any cross-section. Let the eigenstrains again be assumed biaxial and the depth-averaged eigenstrain $\Gamma(r)$ (which now depends on the radial coordinate) be introduced. Let the function $\Gamma(r)$ have bounded support, i.e. require that $\Gamma(r)=0$ for all $r>a$. This implies that the eigenstrain is present only within a circular region of radius $a$ and is zero outside.

Equation (12), upon substitution of the above and some rearrangement, then assumes the form

$$
\frac{\mathrm{d}}{\mathrm{d} r}\left[\nabla^{2} \phi+E \Gamma(r)\right]=0
$$

This can be integrated to give

$$
\phi(r)=B \log \frac{r}{a}-E \int_{r}^{\infty} \frac{1}{r}\left[\int_{r}^{\infty} r \Gamma(r) \mathrm{d} r\right] \mathrm{d} r
$$

so that the average stresses are

$$
\begin{aligned}
& S_{r}=\frac{B}{r^{2}}+\frac{E}{r^{2}} \int_{r}^{\infty} r \Gamma(r) \mathrm{d} r \\
& S_{\theta}=-\frac{B}{r^{2}}-\frac{E}{r^{2}} \int_{r}^{\infty} r \Gamma(r) \mathrm{d} r-E \Gamma(r)
\end{aligned}
$$

Since stresses remain finite as $r \rightarrow 0$, then

$$
B=-E \int_{r}^{\infty} r \Gamma(r) \mathrm{d} r
$$

Note also that stresses vanish at infinitely large distances from the inclusion (where $\Gamma=0$ ).

Further progress can be made if a specific distribution of eigenstrains is chosen. Consider, for example, a circular inclusion with uniform dilatational eigenstrain, i.e. $\Gamma(r)=\Gamma_{0} H(a-r)$, where $H(x)$ denotes the Heaviside step function. In this case $\phi(r)$ is a continuous function, $\phi=\left(E \Gamma_{0} / 4\right)\left(a^{2}-r^{2}\right), \quad r \leqslant a$, and $\phi=\left(-E \Gamma_{0} a^{2} / 2\right) \log (r / a), \quad r>a$. The average stresses are $S_{\theta}=S_{r}=-E \Gamma_{0} / 2, \quad r \leqslant a, \quad$ and $S_{\theta}=-S_{r}=E \Gamma_{0} a^{2} /\left(2 r^{2}\right), r>a$, i.e. for positive eigenstrain the radial and hoop stresses are compressive and equal in the peened area and decay to zero at infinity outside this domain. The radial stress varies continuously across the interface $r=a$, while the hoop stress changes abruptly from compressive to tensile. It is worth noting that the solution for average stresses is identical to the stress field around a circular inclusion in a plane problem.

If the plate deflection is assumed small and the associated stresses disregarded, the through-thickness variation of residual stress in the peened area can now be found by superposing uniformly distributed 
average stresses on the stress profile determined in the previous section. It is clear from the above solution that the magnitude of the near-surface compressive stresses due to peening is increased in the case of local treatment compared to the case of uniform eigenstrain. This effect is associated with the constraint exerted on the peened region by the surrounding material.

If the plate deflection is small, but non-negligible, it must be determined from the equation discussed in the next section.

\section{PLATE DEFLECTION}

Once the stress function $\phi(r)$ has been determined, the deformed shape of the plate (i.e. the out-ofplane displacement of the median plane) can be found by solving the equation for deflection of flexible plates, due to von Karman [17]

$$
\begin{aligned}
\nabla^{2} \nabla^{2} w & =\frac{1}{r} \frac{\mathrm{d}}{\mathrm{d} r}\left\{r \frac{\mathrm{d}}{\mathrm{d} r}\left[\frac{1}{r} \frac{\mathrm{d}}{\mathrm{d} r}\left(r \frac{\mathrm{d} w}{\mathrm{~d} r}\right)\right]\right\} \\
& =-\frac{h}{D r} \frac{\mathrm{d}}{\mathrm{d} r}\left(\frac{\mathrm{d} \phi}{\mathrm{d} r} \frac{\mathrm{d} w}{\mathrm{~d} r}\right)
\end{aligned}
$$

where $D=E h^{3} /\left[12\left(1-\nu^{2}\right)\right]$ denotes the plate bending stiffness. The dimensionless parameters $\rho=r / a$ and $\alpha^{2}=E \Gamma_{0} a^{2} h /(2 D)=6 \Gamma_{0}\left(1-\nu^{2}\right) a^{2} / h^{2}$ can be introduced. Using the solution for the stress function found in the previous section, the deflection equation is obtained in the form

$$
\nabla_{\rho}^{2} \nabla_{\rho}^{2} w= \begin{cases}\frac{\alpha^{2}}{\rho} \frac{\mathrm{d}}{\mathrm{d} \rho}\left(\rho \frac{\mathrm{d} w}{\mathrm{~d} \rho}\right), & \rho \leqslant 1 \\ \frac{\alpha^{2}}{\rho} \frac{\mathrm{d}}{\mathrm{d} \rho}\left(\frac{1}{\rho} \frac{\mathrm{d} w}{\mathrm{~d} \rho}\right), & \rho>1\end{cases}
$$

Via the substitution $v=\rho w^{\prime}$, this equation is transformed to become

$$
v^{\prime \prime}-\frac{v}{\rho}-\alpha^{2} \frac{v}{\rho^{n}}=C_{n}
$$

where $n=0$ for $\rho \leqslant 1$ and $n=2$ for $\rho>1$. By assuming smooth behaviour of the deflection $w(r)$ at the origin and at large values of $r$ it is easily demonstrated that $C_{n}=0$ must be chosen in the present case.

For $\rho<1$, equation (17) is of the modified Bessel type with the general solution given by a linear combination $v(\rho)=A \rho I_{1}(\rho)+B \rho K_{1}(\rho)$, where the functions $I_{n}(\rho)$ are called Bessel functions of imaginary argument and $K_{n}(\rho)$ the Macdonald functions [18].

For $\rho>1$, equation (17) admits simple solutions given by the powers $\rho^{m}, m_{1,2}=1 \pm \sqrt{1+\alpha^{2}}$. Taking into account the asymptotic behaviour of the solutions at small and large values of the arguments [18] and the conditions of continuity for the slope $w^{\prime}$ at $\rho=0$ and $\rho=1$, unknown constants in the general solution for $v=\rho w^{\prime}$ can be eliminated. The solution for the deflection $w$ can then be obtained by integrating the following

$$
w^{\prime}(\rho)= \begin{cases}\frac{A I_{1}(\alpha \rho)}{I_{1}(\alpha)}, & \rho \leqslant 1 \\ A \rho^{-\sqrt{1+\alpha^{2}}}, & \rho>1\end{cases}
$$

with the boundary condition $w=0$ for $\rho \rightarrow \infty$. This gives

$w(\rho)= \begin{cases}w_{0}\left[1+\left(\sqrt{1+\alpha^{2}}-1\right) \frac{I_{0}(\alpha)-I_{0}(\alpha \rho)}{\alpha I_{1}(\alpha)}\right], & \rho \leqslant 1 \\ w_{0} \rho^{-\left[\sqrt{1+\alpha^{2}}-1\right]}, & \rho>1\end{cases}$

where $w_{0}$ denotes the plate deflection at the edge of the peened region, $\rho=1$.

\section{CONCLUSIONS}

The solution has been presented of the axisymmetric problem about elastic deformation of a flexible plate due to a prescribed distribution of eigenstrains, such as may be generated during shot peening treatment. The spatial variation of residual stresses and strains through the plate thickness, and the deformed plate shape, can be predicted using this technique. Important parameters that govern the residual stress distribution, the depth-average eigenstrain and the eigenstrain moment, have been identified.

The framework presented may serve to improve the amount of control exercised in shot peening and peen forming operations.

\section{REFERENCES}

1 Koster, W. P., Gatto, L. R., and Cammett, J. T. Influence of shot peening on surface integrity of some machined aerospace materials. In 1st International Conference on Shot Peening, Paris, 1981, pp. 287-295.

2 Wohlfahrt, H. Shot peening and residual stresses. In Proceedings of the Sagamore Army Materials Research Conference, 1982, pp. 71-92 (Plenum Press, New York).

3 Guechichi, H., Castex, L., Frelat, J., and Inglebert, G. Predicting residual stress due to shot peening. In Impact surface treatment, 1986, pp. 11-22 (Elsevier, London).

4 Korsunsky, A. M. An analysis of residual stresses and strains in shot peening. In Proceedings of the 5th International Conference on Residual stresses, Linkoping, Sweden, 1997.

5 Al-Hassani, S. T. S. Mechanical aspects of residual stress development in shot peening. In 1st International Conference on Shot Peening, Paris, 1981, pp. 14-17. 
6 Kobayashi, M., Matsui, T., and Murakami, Y. Mechanism of creation of compressive residual stress by shot peening. Int. J. Fatigue, 1998, 20, 351-357.

7 Blodgett, M. P. and Nagy, P. B. Eddy current assessment of near-surface residual stress in shot-peened nickelbase superalloys. J. Nondestructive Evaluation, 2004, 23, 107-123.

8 Hauk, V. Structural and residual stress analysis by nondestructive methods, 1997 (Elsevier, Amsterdam).

9 Prevey, P. S. X-ray diffraction characterization of residual stresses produced by shot peening. In Shot peening theory and application (Series Ed. A. NikuLari), IITT-International, Gournay-Sur-Marne, France, 1990, pp. 81-93.

10 Wang, T. and Platts, M. J. A computer-aided blank design for the peen forming process. J. Mater. Processing Technol., 2002, 122, 374-380.

11 Levers, A. and Prior, A. Finite element analysis of shot peening. J. Mater. Processing Technol., 1998, 80-81, 304-308.
12 Guagliano, M. and Vergani, L. An approach for prediction of fatigue strength of shot peened components. Engng Fract. Mechanics, 2004, 71, 501-512.

13 Mura, T. Micromechanics of defects in solids, 1982 (Martinus Nijhoff, Dordrecht).

14 Eshelby, J. D. The determination of the elastic field of an ellipsoidal inclusion, and related problems. Proc. R. Soc., 1957, A241, 376-396.

15 Faivre, G. Déformations de cohérence d'un précipite quadratique. Physica Stat. Sol., 1964, 35, 249-259.

16 Korsunsky, A. M. An axisymmetric inclusion in one of two perfectly bonded dissimilar elastic half spaces. Trans. ASME, J. Appl. Mechanics, 1997, 64, 697-700.

17 Szilard, R. Theory and analysis of plates, 1974 (PrenticeHall, Englewood Cliffs, New Jersey).

18 Gradshteyn, I. S. and Ryzhik, I. M. Table of integrals, series and products, 1965 (Academic Press, New York). 